\title{
Reflexiones sobre la enseñanza y la investigación tecnológica para la vivienda de las mayorías
}

\section{Julián Salas*}

\section{Resumen}

El trabajo pretende un acercamiento inicial a los contenidos y formas de abordar la enseñanza de la arquitectura y la edificación desde el primer mundo, para paliar las necesidades de las "mayorías", o lo que es equivalente, los peligros de la enseñanza desde los enfoques de las "minorías" cuando se intentan transferir a los países en desarrollo. El autor aborda algunos aspectos cruciales de la tecnología pertinente en sus aplicaciones a los problemas del hábitat de "los sin vivienda", propugnando que dicha tecnología: debe comprometerse con la "necesidad"; precisa de investigación específica para la resolución de problemas acuciantes (“investigación-acción”); y debe permitir la libre utilización de sus resultados. Finaliza el trabajo comentando algunos aspectos del Subprograma CYTED XIV: Tecnologías para viviendas de interés social, aún válidos, según el autor, pese a los lustros transcurridos desde su inicio.

\section{Palabras clave}

Vivienda de bajo coste; Investigación-acción; Tecnología apropiable; Docencia apropiada; Transferencia de resultados.

\section{Abstract: Think on the education and the technological investigation for the hou- sing of the majorities}

This paper aims to bring together the contents and forms of approaching the teaching of architecture and construction from the point of view of the first world, to mitigate the needs of the "majority"; or what is equivalent, the dangers of teaching from the point of view of the "minorities" when they try to transfer their approach to the developing countries. The author tackles some crucial aspects on the application of technology to the habitat issues of the "homeless", proposing that this technology: must be committed with the "necessity"; requires specific research for urgent problems' solution ("research-action"); and must allow freely use of its results. He finalises its work commenting some aspects of the CYTED XIV Subprogram: Technologies for social interest housing, still applicable in spite of the time passed since it started.

\section{Key words}

Low cost housing; Action-Research; Appropriable Technology; Appropriate Teaching; Results Transfer.

\footnotetext{
Recibido 23/09/2010; aceptado: 29/11/2010

* Ingeniero industrial, Director de la Cátedra UNESCO en Habitabilidad Básica de la Universidad Politécnica de Madrid, Escuela Técnica Superior de Arquitectura. E-mail: julian.salas@ietcc.csic.es.
} 


\section{Por una enseñanza de la arquitectura para la humanidad}

La arquitectura dominante, la que se enseña en nuestras escuelas y facultades del llamado Primer Mundo, se conforma a partir de fenómenos observados en países "desarrollados" que cuantitativamente constituyen el "caso particular", por lo que resulta intrínsecamente inaplicable al "caso general", si realmente se pretenden paliar necesidades fundamentales de la humanidad, que son preponderantes en los países "subdesarrollados".

El axioma anterior sintetiza el alegato que sigue en pro de una enseñanza diferenciada, así como de la necesidad de generar y difundir conocimientos desde el "caso general" para las necesidades cosmopolitas. No es plausible la consecución de principios y leyes aplicables a todos y en todo lugar, por no ser legítimo construir una teoría general sobre casos particulares.

Hacemos nuestra una premisa que, por obvia, no necesita defensa: son muchos más los habitantes en países catalogados como subdesarrollados que en los desarrollados. Son más los que ni se benefician ni gozan de la arquitectura que los que habitan viviendas en las que hayan intervenido -aun en la forma más elemental imaginable- profesionales de la arquitectura. Naciones Unidas para el Hábitat recuerda que: "Hoy, por cada habitante del mundo desarrollado que vive en ciudades, dos lo hacen en ciudades del Tercer Mundo, y para el año 2030 esta proporción se duplicará, será de cuatro a uno".

Que la arquitectura dominante se gesta y pretende dar respuestas al "caso particular", no cabe duda. También el urbanismo, la construcción, las estructuras, las instalaciones... conforman sus propuestas desde el "caso particular". Que éstas no se aplican al "caso general", puede palparse en la realidad formal y espacial de las periferias y barrios marginales del Tercer Mundo, que cobijan a más de la mitad de la humanidad. Por ello, gran parte de los profesionales de la arquitectura no se reconocen en este tipo de soluciones habitacionales. Las califican de "indignas", para dejar claro que no son resultado de su profesión y ven en ellas la razón del "problema" en lugar de las semillas de la "solución".

¿Imaginan como materia académica unos "Principios de Astronomía" que únicamente tratasen de la Tierra o incluso sólo del sistema solar? La evidencia material y espacial invalida extrapolar del "caso particular" al "caso general". Ocurre al enseñar economía, ingeniería, medicina, agricultura... y lamentablemente no son legión los profesionales verdaderamente conscientes de las barreras que el origen occidental-desarrollado impone a su ciencia, técnica o profesión.

Jorge Hardoy, en su trabajo Repensando la Ciudad del Tercer Mundo, afirmaba:

"A pesar de que en la actualidad el mundo cuenta con más profesionales arquitectos, planificadores e ingenieros capacitados para trabajar en áreas urbanas que nunca antes en la historia, esos profesionales tienen poca o ninguna repercusión en el mejoramiento de las condiciones de los grupos de menores ingresos" (Hardoy, 1985).

Esos profesionales y los colegios o instituciones que los representan -también en el Tercer Mundo, lo que es más preocupante- se muestran renuentes a enfrentarse a sus nuevos roles. Existen tareas fundamentales, que asumen en parte los nuevos profesionales en el sector informal, en instituciones de la sociedad civil organizada o de cooperación para el desarrollo, consistentes en asesorar y trabajar con grupos populares; son los otros profesionales a los que el sector informal reconoce como actores importantes de dinamización de la construcción y el desarrollo de la ciudad.

Por otra parte, los gobiernos locales requieren con insistencia administradores y planificadores urbanos con capacidad para trabajar y negociar con la sociedad organizada, comprometidos en "la construcción de abajo hacia arriba". Se tiene constan- 
cia de lo mucho que, en Latinoamérica especialmente, han realizado en las últimas décadas los profesionales involucrados en las organizaciones sociales: han diseñado viviendas, caminos, calles y áreas de juego; han instalado agua potable, energía eléctrica y alcantarillado; han revitalizado barrios; han hecho frente a las emergencias por terremotos, inundaciones, ciclones...; han articulado respuestas durante graves crisis económicas y conflictos armados; han impartido cursos y proporcionado formación profesional; han formulado propuestas y estudios de desarrollo; han ofrecido habitabilidad básica a los invasores de terrenos; han apoyado el tejido productivo barrial; han planteado y difundido problemas y soluciones factibles para la ciudad; han creado espacios de diálogo y negociación con las administraciones públicas; han diseñado y ejecutado proyectos sociales con financiación nacional, internacional y multilateral...

De la toma de conciencia de la situación que se comenta, a actuar en consecuencia, hasta sumergirse en un proceso de "desaprendizaje de la profesión", en el que se afanó John F. Turner al encontrarse de frentón con la realidad de las invasiones de tierras por autoconstructores peruanos en la década de los sesenta, media un largo trecho rico en matices. Pese a la radicalidad de las propuestas de Turner, nos sigue pareciendo recomendable en este contexto la lectura de su obra, aunque nos encontremos más cerca de Víctor Pelli cuando afirma que:

"(...) si bien puede ser discutible si es o no Arquitectura lo que se construye y lo que se hace para resolver la pobreza habitacional, de lo que no hay duda es de que en este trabajo -en el caso general- hacen falta arquitectos" (Pelli).

Arquitectos que trabajen para paliar las necesidades más perentorias que sintetizamos como "habitabilidad básica", capaz de colmar las carencias esenciales de cobijo que tenemos todas las personas, lo que constituye el eje vertebrador de la formación que impartimos en la Escuela de Arqui- tectura de Madrid ${ }^{1}$.

Si el lector ha llegado hasta aquí, perdonará el silencio sobre nuestra fuente inspiradora. Leyendo a Dudley Seers, director del Instituto de Estudios para el Desarrollo de Sussex, Inglaterra, nos impactó su trabajo The Limitations of the Special Case en el que afirma:

“(...) la economía dominante, enseñada en las universidades, se construye a partir de fenómenos observados en los países «desarrollados» (los que constituyen precisamente el «caso particular»), por lo que, en consecuencia, es inaplicable al caso general, el de los países "subdesarrollados»" (Seers, 1963).

La crítica de Seers ponía en cuestión demasiadas cosas para que fuesen atendidas todas, pese a que lo que implícitamente proponía hace cinco décadas no era otra cosa que darle carta de naturaleza a una nueva disciplina: la "Economía del Desarrollo", una apostasía académica entonces y una disciplina curricular clásica hoy.

\section{Por una tecnología comprometida con la necesidad}

La industrialización "posible" de la vivienda latinoamericana (Salas, 2000), que pretendió ser una reflexión, documentada y realizada sobre el terreno, aspiraba a transferir ideas y soluciones del Norte al Sur y fundamentalmente del Sur al Sur, defendiendo, que para afrontar soluciones de vivienda de muy bajo presupuesto en Latinoamérica hay que evitar que la inteligencia y la voluntad se ahoguen en océanos de datos y teorías que explican demasiado y que esclarecen tanto como aprisionan en algún estrecho corsé interpretativo. Creímos que describir herramientas y realizaciones eficaces podría revelarse como un ejercicio sinérgico de interés. Nos vimos abocados a este recurso, en parte, ya que las teorías sobre la in-

\footnotetext{
1 Cursos de Especialización de la Universidad Politécnica de Madrid (ETSAM) en Cooperación para el Desarrollo de Asentamientos Humanos en el Tercer Mundo, que se encuentran en su XIV edición: <http://www.cuhabupm.es/>.
} 
dustrialización de la vivienda posible ni abundan, ni han abierto caminos fructíferos en los últimos tiempos. Frente a resultados insatisfactorios de sus estrategias, en ocasiones desastrosas, los técnicos hemos esgrimido coartadas que van desde el carácter impredecible de la realidad a la supuesta o real pusilanimidad de los políticos, que, en opinión de los técnicos, se asustan ante dosis prescritas de remedios, naturalmente amargos.

En el ámbito de las técnicas constructivas para la vivienda de interés social, Latinoamérica vive un gran parón, una especie de calma chicha en la aportación de ideas, alternativas, soluciones tangibles...; una especie de boicot de ofertas para una demanda gigantesca, solvente pero de bajo poder adquisitivo. Decía cantando Mercedes Sosa que no corren buenos tiempos para la poesía; tampoco para la prosa, añadiríamos nosotros. En esta atonía generalizada de la que sólo emergen algunos profesionales e instituciones singulares, sólo: el convencimiento obstinado que proporciona el contacto in situ con la magnitud; el dramatismo de la necesidad; el haber palpado encomiables resultados de "industrialización posible" y conocido fracasos de soluciones tecnológicas que no repetiríamos, hicieron que nos mantuviésemos perseverantes en el empeño de alumbrar el trabajo mencionado (Salas, 2000), que pretendía ofrecer tecnologías libres -en el sentido de no estar hipotecadas por patentes- y comprometidas con la necesidad.

La propuesta que sostenemos en La industrialización "posible" de la vivienda latinoamericana coincide en parte, sólo en parte, con Ugo Pipitone cuando afirma:

"El subdesarrollo es un castillo que no se rinde ante un largo asedio, sino sólo por asalto; o sea, en tiempos históricos restringidos. Obviamente, 40 ó 50 años pueden parecer mucho tiempo en la vida de un individuo; en la historia de las naciones es, generalmente, poco más que un parpadeo" (Pipitone, 1999).
Nuestra matización a esta reflexión consistiría en que para estos supuestos 40 ó 50 años, mientras que el Tercer Mundo recorre el largo, tortuoso e incierto camino tecnológico hasta el hipotético asalto a la "industrialización sutil" (Salas, 1997), no vislumbramos nada tan útil y tangible, con todas nuestras dudas, como la "industrialización posible". En un contexto de necesidades vitales abrumadoras optamos por ser contemporáneos antes que modernos.

\section{CYTED 2 : investigación tecnológica pa- ra la vivienda de los "sin vivienda"}

\section{Vigencia de una propuesta de hace cinco lustros...}

Durante 1986, y siguiendo la metodología del Programa Ciencia y Tecnología para el Desarrollo (CYTED, 1987), se elaboró el documento de bases del Subprograma XIV: Tecnologías para Viviendas de Interés Social, tras visitar 86 centros de investigación-acción de 14 países latinoamericanos, realizar 173 entrevistas documentadas y recoger la reseña de 254 áreas temáticas de investigación. Finalmente, se estructuró la propuesta (ver Figura 1) en forma de cuatro tomos y un total de 1.200 páginas con los siguientes capítulos:

- Introducción y acotaciones a la tecnología para viviendas de interés social.

- Datos y aportaciones relevantes sobre el tema.

- Reseña de instituciones y proyectos vigentes en Latinoamérica.

- Propuesta de estructuración del Subprograma CYTED XIV: Tecnologías para viviendas de interés social.

\footnotetext{
${ }^{2}$ Nos referiremos en todo momento al Programa CYTED, en lo que podíamos denominar Primera Época -entre su creación en 1985 y 1995- en la que no se camuflaba o escondía la "D" del desarrollo, ya que, de ser su objetivo baluarte la aplicabilidad de sus resultados al "desarrollo" de Latinoamérica, ha pasado a considerar este objetivo como un lastre para la consecución de pretender resultados "competitivos y de excelencia internacional".
} 


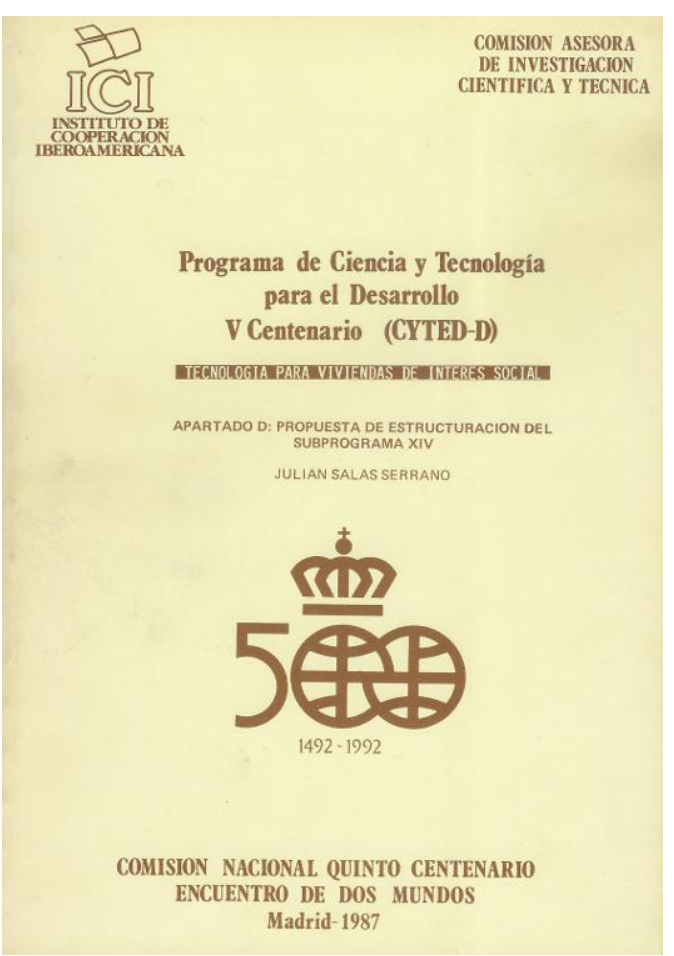

Figura 1: Portada del Informe de Estructuración del Subprograma CYTED. XIV: Tecnologías para viviendas de interés social.

El documento finalizaba con un capítulo de propuestas, que más adelante reproduciremos textualmente -ya que pese a los lustros transcurridos entendemos que prevalece gran parte de su interés. Las conclusiones de ese arduo trabajo se ordenaron en forma de los siguientes apartados:

a. Justificación de una prioridad previa;

b. Propuesta de elaboración de dos estudios de apoyo al Subprograma XIV;

c. Estructuración del Subprograma: siete proyectos de investigación.

\section{Una prioridad previa}

Los centros de investigación y desarrollo y, más ampliamente, el conjunto de la infraestructura de I+D que se ocupan en Iberoamérica de tareas relacionadas con "tecnología para vivienda de interés social" muestran, en general, una gran debilidad estructural, concluíamos entonces, y nos aventuramos a pensar que se han deteriorado aún más hoy. Por otra parte, los escasos recursos económicos destinados a apoyar actividades científico-tecnológicas en estos temas no cuentan, sal- vo excepciones, con programas financiados que racionalicen el esfuerzo y coadyuven a resolver la gravísima problemática de vivienda existente.

Coincidiendo con la recomendación -que era simultánea en el momento de la propuesta- de la Cuarta Conferencia General de ONUDI "(...) el fortalecimiento de las capacidades tecnológicas es requisito para la adquisición y la aplicación de tecnología importada y para el desarrollo de tecnología del país"-, se propuso y ratificaríamos hoy como prioridad global, la necesidad de: "crear, consolidar y/o reforzar las capacidades científicas y tecnológicas existentes en tecnología para viviendas de interés social, ya que se estima como una tarea de la más alta prioridad".

\section{Dos propuesta de estudios de apoyo al Subprograma CYTED XIV}

La temática del Subprograma presenta una serie de particularidades que se trataron de poner de manifiesto en el documento al que nos estamos refiriendo. En base a las características e implicaciones socioeconómicas del tema, se proponía acometer el estudio y elaboración de los siguientes trabajos aplicados, de apoyo al Subprograma XIV, que nos siguen pareciendo urgentes:

- Diseño de nuevas fórmulas y/o adecuación de los sistemas de financiación de viviendas de interés social a las particularidades constructivas del Área: autoconstrucción, ayuda-mutua, sitio-servicios, asentamientos espontáneos, otras.

- Programa para la formación de profesionales a todos los niveles en la especialidad de tecnologías para viviendas de interés social y asentamientos populares (programa que hoy explicitaríamos como enseñanza a varios niveles para la dotación de "Habitabilidad Básica" y para la "Mejora de Tugurios").

\section{Siete proyectos de investigación-acción}

La propuesta final de estructuración del Subpro- 
grama XIV se concretó en la recomendación de abordar la puesta en funcionamiento paulatino de siete proyectos, que, en opinión del autor, cumplían en forma simultánea dos características: ser social y económicamente necesarios; contar en el Área con masa crítica de personal y conocimientos suficientes y la posibilidad fundada de obtener recursos para su desarrollo.

Los proyectos seleccionados fueron:

XIV.A. Tecnologías para la construcción a base de madera: caracterización de maderas; optimación de su empleo (durabilidad y pirorresistencia); soluciones constructivas a base de componentes de madera.

XIV.B. Elementos, componentes y sistemas constructivos para su empleo en viviendas crecederas y/o mejorables mediante autoconstrucción o ayuda mutua: en situación urbana, en el ámbito rural.

XIV.C. Tecnologías para la construcción a base de tierra: optimización del empleo de tierra (resistencia, comportamiento ante la humedad, tratamiento de paramentos, etc.). Soluciones específicas constructivas y estructurales para su utilización en zonas con alta sismicidad potencial.

XIV.D. Desarrollo, experimentación y evaluación de tecnologías apropiadas para la mejora y consolidación de asentamientos populares espontáneos. Construcción y valorización de soluciones habitacionales y servicios comunitarios.

XIV.E. Desarrollo de acuerdos de compatibilización, coordinación y de prestaciones funcionales de elementos, subsistemas y sistemas constructivos, para viviendas de interés social, mediante tecnología libre a base de elementos prefabricados y utilizando la capacidad instalada en el área. XIV.F. Soluciones de cubiertas a base de elementos ligeros realizados prioritariamente mediante materiales y/o subproductos locales capaces de sustituir las placas usuales (asbesto-cemento o zinc).

XIV.G. Optimización de procesos de producción y de empleo del cemento portland. Caracterización tecnológica de adiciones y de hormigones a base de cementos con adiciones.

(Propuestas elaboradas en diciembre de 1986).

En el Subprograma Tecnologías para viviendas de interés social en Latinoamérica participaron 112 grupos de 17 países y fue en opinión de la CEPAL “(...) el mayor esfuerzo de investigación-acción jamás realizado en materia de hábitat popular en América Latina y el Caribe".

\section{Transferencia de tecnología en el sec- tor del hábitat popular}

\section{Acotaciones a la tecnología pertinente}

El tema merece algunas acotaciones previas que se estiman pertinentes sobre qué entender por tecnología en este contexto. Sin entrar en mayores matizaciones, ofrecemos como definición de tecnología la propuesta por Ortega y Gasset:

"La técnica es el esfuerzo para ahorrar esfuerzo. Aquello a lo que dedicamos el esfuerzo a inventar y ejecutar un plan para: asegurar la satisfacción de las necesidades elementales; lograrlo con el mínimo esfuerzo; crear objetos que no hay en la naturaleza y caminar con ella -con la técnica- hacía la vida buena y la emancipación humana". Y terminaba Ortega ratificando que la técnica "[...] debe de estar siempre al servicio de lo propiamente humano" (Ortega y Gasset, 1952).

La definición anterior se materializa cabalmente, a modo de ejemplo, en los aportes del trabajo desarrollado durante décadas por el colombiano Álvaro Ortega, aunando de forma magistral tecnología y función social de la arquitectura, y que personalmente concretaría en el legado universal de las placas de techo "gran onda, teja canaleta o canalonda", resultado de su trabajo de tesis doctoral en Harvard (1945) con Walter Gropius. Trabajos recogidos en el libro A/varo Ortega. Prearquitecturas del bienestar (Ortega, 1998), injustamente ignorado en Latinoamérica (véanse Figuras 2 y 3 ). 


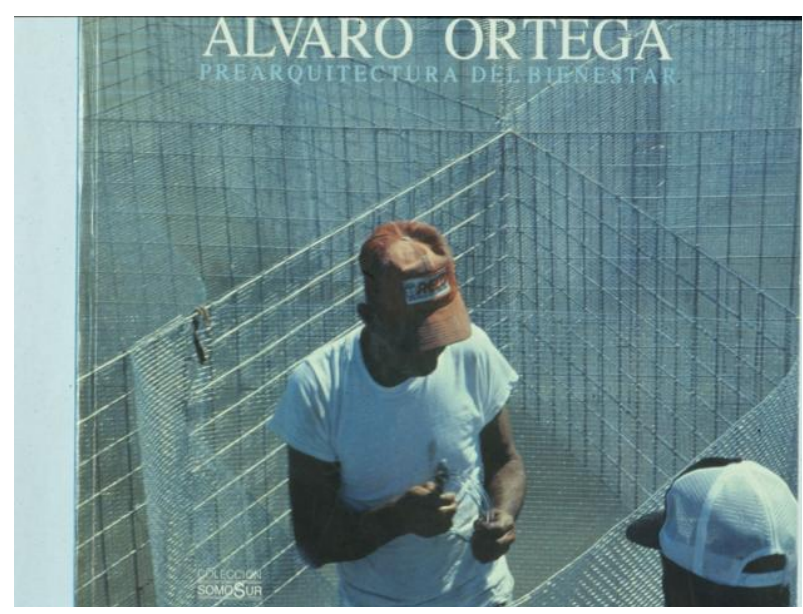

Figura 2: Portada del libro sobre la obra de A. Ortega, editado por la Colección SOMOS-SUR de la Editorial Escala de Colombia (1998).

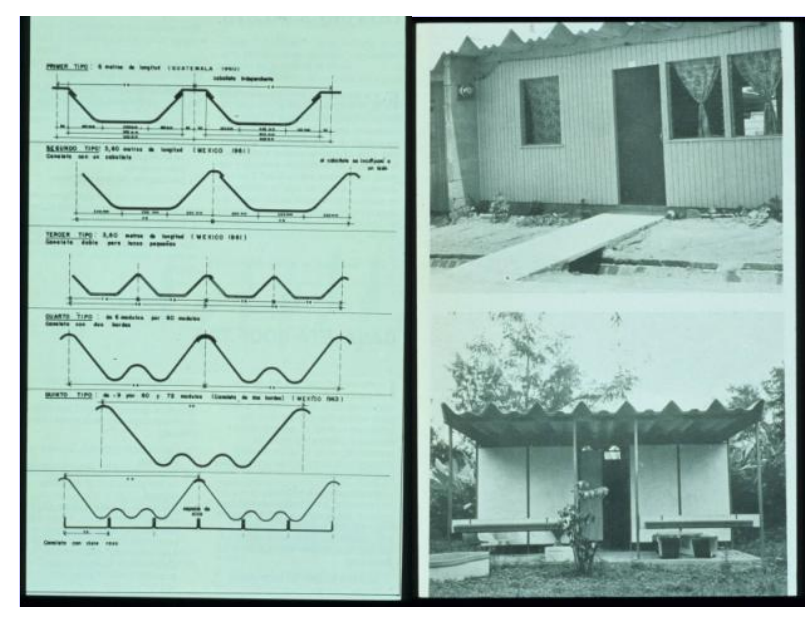

Figura 3: llustraciones en el libro mencionado (de A. Ortega), de los principios que inspiraron el trabajo para incrementar el canto de la sección transversal de las placas al objeto de eliminar la estructura portante en las viviendas de muy bajo coste.

Igualmente notables nos parecen las aportaciones del arquitecto brasileño João Filgueiras Lima, "Lelé" (Salas, 2000), generoso en tenacidad y genio creador, que llegó a construir más de doscientas escuelas prefabricadas en Río de Janeiro por año entre 1984 y 1988 y que recientemente "producía" hospitales bellos y funcionales en el Centro de Tecnología da Rede SARAH de Salvador de Bahía. Esta auténtica fábrica de vanguardia, con capacidad para construir anualmente hospitales para recibir hasta cuatrocientas nuevas camas, empresa difícilmente concebible en el Primer Mundo y aún más impensable en las coordenadas de Brasil.

\section{El concepto de transferencia en este ámbi- to}

Existen multitud de definiciones de lo que es o no transferencia tecnológica; su bondad depende del contexto y circunstancias en las que han de utilizarse. El ámbito al que se circunscribe este trabajo es nítido: el hábitat popular en su acepción más amplia, abarcando, en cualquier caso, tanto los productos (físicos, tangibles) como los procesos utilizados para su consecución (organizativos, sociales, de formación...), así como la transferencia y difusión de procesos innovadores y/o de los productos imprescindibles.

No entendemos la tecnología como la define el Diccionario de la Real Academia de la Lengua Española: “( ...) conjunto de conocimientos propios de un oficio mecánico o arte industrial". Tampoco nos identificamos con los que la consideran como conjunto de máquinas, herramientas o equipos materiales -lo que llamaríamos hoy hardware-, pese a las connotaciones de prestigio, modernidad y progreso que suele concitar este posicionamiento en el sector de producción de viviendas. Valoramos muy positivamente como tecnología del hábitat popular, aspectos de organización de obra, planificación integral, racionalización de procesos, programación de suministros... aspectos blandos de la tecnología, que en su conjunto se acerca a lo que se entiende como software, para los que preferimos acuñar un nuevo barbarismo: "ordware".

El "ordware" no es otra cosa que la materialización del orden en lo general y en lo particular, en lo grande y en los detalles. El orden como fuente de racionalización de tareas, minimización de desechos, coordinación de oficios... No es una meta fácil en el mundo de la construcción. Para que el personal y los equipos funcionen al unísono, hay que poner los medios y no desfallecer. Se necesitan: puestos de trabajo estables, formación profesional continua, órdenes escritas y precisas, salarios dignos... Asignamos una gran im- 
portancia al "ordware" en los procesos de racionalización del hábitat por la escasa inversión monetaria que requiere en relación a la altísima rentabilidad que puede obtenerse de su aplicación. El "ordware" nos parece un primer paso obligado para alcanzar y transitar por la industrialización posible (Salas, 2000).

Como síntesis de lo anterior, y sin llegar a formularla como definición cerrada, entendemos por tecnología habitacional la "combinación de procesos, materiales, equipos y conocimientos destinados a la producción de viviendas".

Aceptamos, sin entusiasmo, la expresión transferencia tecnológica. Estaríamos más conformes con denominarla pura y llanamente: compra-venta de tecnología. En cualquier caso, el mercado de tecnologías nace de la desigualdad existente entre los que la poseen respecto de los que no la tienen. La transferencia surge mayoritariamente de las diferencias entre países desarrollados y subdesarrollados. Proponemos diferenciar entre transferencia vertical de tecnología, cuando el trasvase se hace desde el ámbito de la teoría al de la práctica (de la universidad, centro de investigación... a la empresa) y transferencia horizontal, cuando se realiza entre diferentes sectores productivos, diferentes países, o, incluso, entre empresas del mismo sector.

Nos parece plenamente vigente en los sectores de producción del hábitat en su sentido más amplio (planeamiento urbano, habitabilidad básica, vivienda, producción de materiales y componentes, transporte, servicios...) el concepto de código genético de las tecnologías, en el sentido que lo acuñó K. Reddy al afirmar:

"que toda opción tecnológica parece disponer de un código genético, de tal forma que cuando en condiciones favorables consigue implantarse dicha tecnología en un nuevo medio, tiende a reproducir las condiciones socioculturales en las que se gestó" (Reddy, 1978).

Rechazamos la denominación tecnología apropia- da por equívoca y conceptualmente nociva. No conocemos tecnología alguna que, en forma genérica y a priori, merezca tal calificativo. Ninguna tecnología merece tal denominación si antes no se ha contrastado su validez e idoneidad en un determinado contexto. En el sector del hábitat popular en Latinoamérica, se ha abusado del empleo de esta denominación. La concurrencia de algunas características consideradas emblemáticas (el empleo intensivo de materiales autóctonos; la utilización de mano de obra semi-voluntaria o sub-remunerada; la participación activa de los usuarios...) ha sido, en no pocos casos, razón suficiente para adjudicarle la categoría de "apropiada" a una tecnología, marginando otros criterios de gran importancia (relación costo/calidad; durabilidad de lo ejecutado; minimización del desperdicio de materiales; facilidad de apropiación; posibilidad de utilización en escalas diversas, etc.).

\section{La necesaria búsqueda de otros materiales y otras aplicaciones}

Si nos referimos al material más cosmopolita y emblemático de la edificación, el cemento, hay que dejar claro que su consumidor mayoritario es el llamado sector informal. La Associaçâo Brasileira de Cimento Portland (ABCP, 2007) asegura que desde 1990 hasta 2006 el consumo de cemento en Brasil ha sido siempre superior en el sector informal -al que llaman sector formiga- que en el formal. Cemex, por su parte, anunciaba en México, en 2007, que:

“(...) la empresa ratifica su compromiso con la autoconstrucción lanzando al mercado su nuevo producto «Cemento Tolteca» en bolsas de 25 kilogramos. Ello hay que enmarcarlo en el reconocimiento del crecimiento del consumo informal de cemento que actualmente supera el $55 \%$ del mercado cementero del país" (Cemex, 2007).

Su Director General, Dimitri Papalexopoulos, Gerente General de "Cementos Titán”, en la reunión internacional de la CEMENT en el año 2008, afir- 
maba:

"[...] No hay nada que hacer, la producción de cemento siempre supondrá emisiones de dióxido de carbono. No se pueden cambiar las leyes de la química, no podemos esperar cambios espectaculares en la disminución de las emisiones. Las compañías productoras estamos nerviosas ante las regulaciones futuras" (Papalexopoulos, 2008).

Tienen sobradas razones para estarlo.

La crucial importancia del consumo de materiales básicos de construcción por los "sin vivienda" queda implícita en los siguientes datos: a los 2.220 millones de toneladas de producción mundial de cemento en 2005, China aportó 1.000 MTn, India 130 MTn y Estados Unidos 99 MTn.

En forma muy concisa y como conclusiones de trabajos realizados en la "Cátedra UNESCO"
(Salas y Oteiza, 2008) puede afirmarse que:

- La repercusión porcentual del capítulo "Construcción" en el precio final de la vivienda es mayor cuanto menor es su presupuesto.

- La repercusión porcentual del capítulo "Materiales" en el total de la "Construcción" aumenta cuanto menor es el presupuesto total y el nivel de desarrollo del país en el que se realice, llegando a valores extremadamente dispares: $13,32 \%$ en el sector formal de los países desarrollados; $31,55 \%$ en el sector formal de Latinoamérica; y 49,00\% en el sector informal del Tercer Mundo (Ver Figura 4).

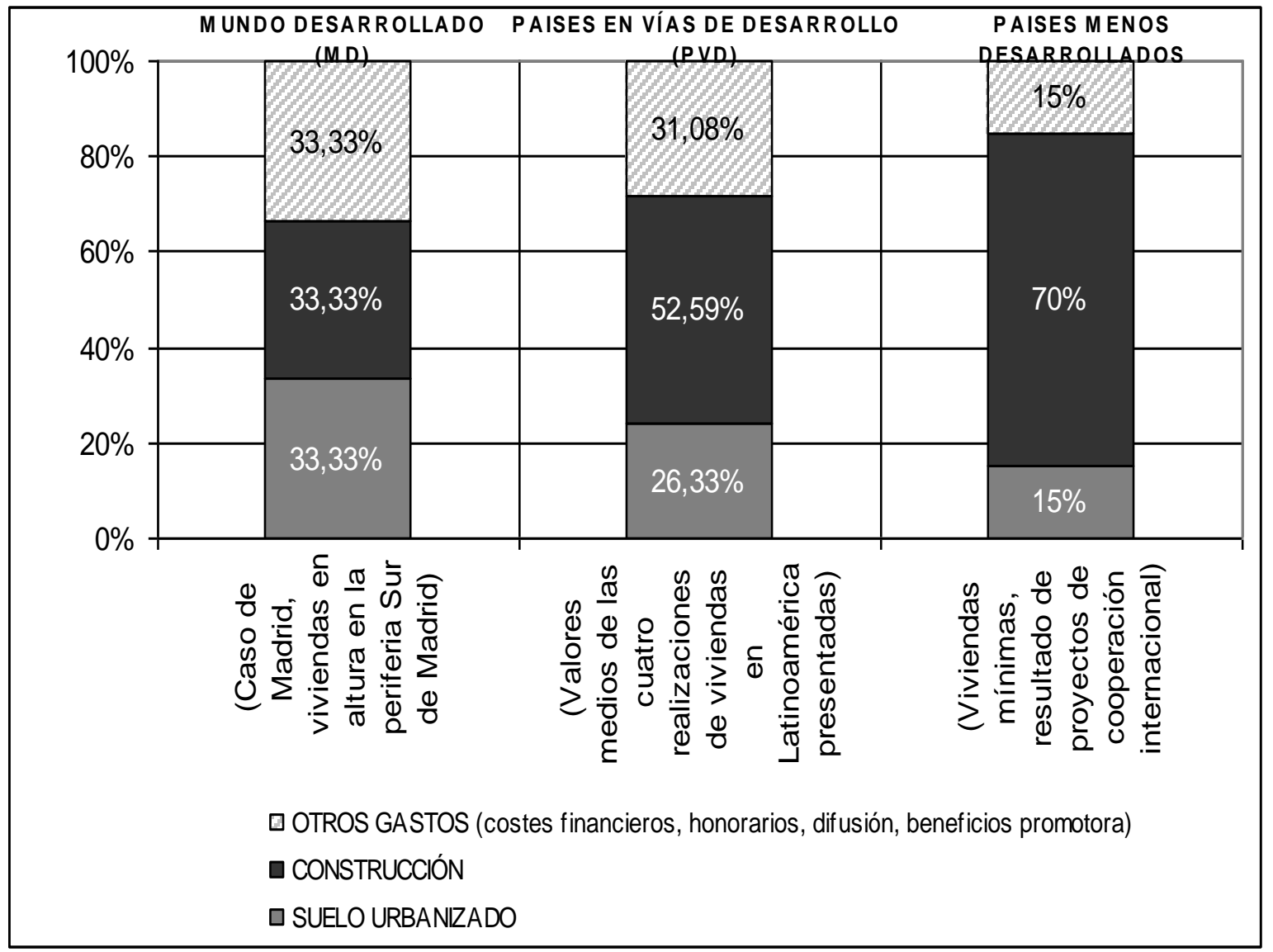

Figura 4: Ejercicio de aproximación a la cuantificación de la repercusión del coste de construcción en el precio final de lo construido en tres supuestos muy diferentes. Fuente: Salas y Oteiza (2008).

Durante la segunda mitad del pasado siglo, y aún hoy, los procesos de industrialización y las crecientes posibilidades de comercio y transporte in- ternacional han permitido que algunos componentes, y en especial las placas de asbesto-cemento y las de zinc, se generalizasen de forma masiva en 
todas las latitudes. Estos componentes de origen industrial, fáciles de transportar, manejar, colocar y sustituir -lo cual no es poco- tienen, además, una alta apreciación social entre los sin recursos, como símbolo de acceso a nuevas formas de cobijo. Desde nuestras coordenadas puede resultar inadmisible para muchos, pero la chapa de zinc, pese a sus graves puntos débiles, que los tiene, sigue siendo una excelente solución de techos elementales.

En este contexto, resulta ejemplarizante la "canaleta" o placa "gran-onda" ya aludida anteriormente, ya que mediante el diseño de su sección transversal se consiguió triplicar su inercia respecto de la placa "onda normal", y por ende, aumentar considerablemente su capacidad resistente a flexión. La canaleta, que vuela entre apoyos siete metros con un canto de tan sólo $30 \mathrm{cms}$. se desarrolló pensando en la vivienda de los "sin recursos", tratando de conseguir un elemento universal de manejo manual, que consiguiese de forma simultánea cubrir luces amplias con elementos de mercado de manejo manual.

Para estimular y mejorar el desarrollo del sector "informal" de materiales de construcción, ONUDI (1995) proponía hace tres lustros, entre otras, las recomendaciones que siguen y que en su momen-

to comentamos en Materiales de Construcción (Salas, 1995):

- Reconocer la importancia de la producción de elementos y materiales de construcción del sector "informal" y prestar pleno apoyo a los proyectos encaminados al desarrollo de este sector.

- Facilitar, en proyectos de construcción estatales, el empleo de materiales de construcción producidos por el sector no estructurado.

- Alentar la aceptación y, en su caso, la elaboración de normas y especificaciones que permitan el empleo de materiales y elementos producidos por el sector "informal" y englobar sis- temáticamente estos materiales y elementos en las reglamentaciones de construcción mediante el concepto de niveles de exigencia.

- Reforzar los vínculos entre las actividades de investigación competitiva de ámbito nacional o internacional con necesidades específicas del sector no estructurado.

- Ayudar a difundir y formular normas y especificaciones para materiales locales.

Pese a los muchos años transcurridos, los autores estiman vigentes en plenitud las recomendaciones de ONUDI, y consideran plausible concitar el debate y la reflexión entre investigadores y técnicos del sector sobre los grandes retos que ha de afrontar la industria de materiales básicos de edificación ante las ingentes necesidades actuales.

\section{Referencias}

ABCP. Memoria de la Associaçâo Brasileira de Cimento Portland. São Paulo, 2007.

CEPAL. Alojar el Desarrollo: Una tarea para los asentamientos humanos. Documento LC/L906. CONF.85/3. In Actas de la Reunión CYTED: Iberoamérica ante Hábitat II. Madrid: Ministerio de Fomento, 1996.

HARDOY Jorge E. Repensando la ciudad del Tercer Mundo. Buenos Aires: IIED-América Latina, 1985.

ORTEGA, Álvaro. Prearquitecturas del bienestar. Bogotá: Escala, 2003.

ORTEGA y GASSET, José. Meditación de la técnica. Charla en Buenos Aires, 1952. Madrid: Alianza, 2000.

PAVITT, Keith \& PATEL, Pari. The international distribution and determinants of technological activities. Oxford Review of Economic Policy, 1998, vol. 4 , no 4, p. 35-55.

PIPITONE, Ugo. Siete argumentos (sin una teoría) para salir del subdesarrollo. Claves de Razón Práctica, 1999, nº 92, p. 40-45.

REDDY, Kumar. Background and Concept of 
Appropiate Technology. Documento UNIDO, Conferencia en India, 1978.

SALAS, Julián y OTEIZA, Ignacio. La industria de materiales básicos de construcción, ante las ingentes necesidades actuales de edificación. Materiales de Construcción, 2008, vol. 58, p. 129148.

SALAS, Julián. CYTED: Programa de Ciencia y Tecnología para el Desarrollo: Subprograma XIV: Tecnologías para Viviendas de Muy Bajo Coste. Cuatro tomos mimeografiados. Madrid: ICI, 1987.

SALAS, Julián. Producción flexible versus producción masiva: arquitectura para grandes necesidades. $a+t, 1997$, № 10, p. 22-33.

SALAS, Julián. Formas de la Necesidad. EL PAÍS,
Babelia. Madrid, 24 de junio de 2000.

SALAS, Julián. La industrialización "posible" de la vivienda latinoamericana. Bogotá: Escala, 2000. SEERS, Dudley. The Limitations of the Special Case. Instituto de Estudios para el Desarrollo de Sussex, 1963.

\section{Cita del artículo}

SALAS, Julián. Reflexiones sobre la enseñanza y la investigación tecnológica para la vivienda de las mayorías. Hábitat y Sociedad, 2010, № 1, p. 121-131. $<$ www.habitatysociedad.us.es $>$.

http://dx.doi.org/10.12795/HabitatySociedad.2010.i1.08 